\title{
The Southern H II Region Discovery Survey (SHRDS): Pilot Survey
}

\author{
C. Brown ${ }^{1}$, C. Jordan ${ }^{2}$, John M. Dickey ${ }^{1}$, L. D. Anderson ${ }^{3,4,5}$, W. P. Armentrout ${ }^{3}$, Dana S. Balser ${ }^{6}$, \\ T. M. Bania ${ }^{7}$, J. R. Dawson ${ }^{8,9}$, N. M. Mc Clure-Griffiths ${ }^{10}$, and Trey V. Wenger ${ }^{6,11}$ \\ ${ }_{1}^{1}$ School of Physical Sciences, Private Bag 37, University of Tasmania, Hobart, TAS, 7001, Australia \\ ${ }^{2}$ International Centre for Radio Astronomy Research, Curtin University, Perth, WA, 6845, Australia \\ ${ }^{3}$ Department of Physics and Astronomy, West Virginia University, P.O. Box 6315, Morgantown, WV 26506, USA \\ ${ }^{4}$ Adjunct Astronomer at the Green Bank Observatory, P.O. Box 2, Green Bank WV 24944, USA \\ ${ }^{5}$ Center for Gravitational Waves and Cosmology, West Virginia University, Chestnut Ridge Research Building, Morgantown, WV 26505, USA \\ ${ }^{6}$ National Radio Astronomy Observatory, 520 Edgemont Road, Charlottesville, VA 22904, USA \\ ${ }^{7}$ Institute for Astrophysical Research, Department of Astronomy, Boston University, 725 Commonwealth Avenue, Boston, MA 02215, USA \\ ${ }^{8}$ Department of Physics and Astronomy and MQ Research Centre in Astronomy, \\ Astrophysics and Astrophotonics, Macquarie University, NSW, 2109, Australia \\ ${ }^{9}$ CSIRO Astronomy and Space Science, P.O. Box 76, Epping, NSW, 1710, Australia \\ ${ }^{10}$ Research School of Astronomy \& Astrophysics, The Australian National University, Canberra ACT 2611, Australia \\ ${ }^{11}$ Department of Astronomy, University of Virginia, P.O. Box 400325, Charlottesville, VA 22904-4325, USA \\ Received 2017 February 26; revised 2017 March 28; accepted 2017 March 30; published 2017 June 23
}

\begin{abstract}
The Southern H II Region Discovery Survey is a survey of the third and fourth quadrants of the Galactic plane that will detect radio recombination line (RRL) and continuum emission at cm-wavelengths from several hundred $\mathrm{H}$ II region candidates using the Australia Telescope Compact Array. The targets for this survey come from the WISE Catalog of Galactic H II Regions and were identified based on mid-infrared and radio continuum emission. In this pilot project, two different configurations of the Compact Array Broad Band receiver and spectrometer system were used for short test observations. The pilot surveys detected RRL emission from 36 of $53 \mathrm{HII}$ region candidates, as well as seven known H II regions that were included for calibration. These 36 recombination line detections confirm that the candidates are true $\mathrm{H}$ II regions and allow us to estimate their distances.
\end{abstract}

Key words: Galaxy: structure - HII regions - radio lines: ISM - surveys

Supporting material: machine-readable table

\section{Introduction}

$\mathrm{H}$ II regions are zones of ionized gas surrounding young ( $\sim 10$ Myr old), massive stars. They are some of the brightest objects in the Galaxy at infrared and radio wavelengths, and so they can be detected across the entire Galactic disk (Anderson et al. 2011). H II regions are the archetypal tracers of Galactic spiral structure, and their chemical abundances provide unique and important probes of billions of years of Galactic chemical evolution (Shaver et al. 1983). They are the main tracer of ionizing photons in the Galaxy and can be used to compute global star formation rates. Unlike most tracers of high-mass star formation (e.g., far-infrared clumps), H II regions unambiguously locate sites where massive stars have recently formed. An unbiased Galaxy-wide sample of $\mathrm{H}$ II regions is required to understand the global properties of the Milky Way and to compare its star formation rate to those of external galaxies.

The H II Region Discovery Survey (HRDS) is a collection of radio recombination line (RRL) and continuum emission surveys between 4 and $11 \mathrm{GHz}$, designed to detect all $\mathrm{H}$ II regions ionized by single or multiple O-stars across the entire Galactic disk. All HRDS component surveys are targeted toward $\mathrm{HII}$ region candidates, selected to have spatially coincident $\sim 25 \mu \mathrm{m}$ mid-infrared and $\sim 20 \mathrm{~cm}$ radio continuum emission, surrounded by $\sim 10 \mu \mathrm{m}$ emission-a basic morphology shared by all Galactic H II regions (Anderson et al. 2014). But these criteria are not sufficiently robust to exclude all other kinds of radio and infrared sources; to confirm that they are $\mathrm{H}$ II regions and to measure their radial velocities, it is necessary to detect RRL emission from each candidate.
The primary instrument used for the HRDS is the Green Bank Telescope (GBT; Bania et al. 2010). Spanning $-16^{\circ}<\ell<67^{\circ}$ and $|b|<1^{\circ}$, the GBT HRDS detected 602 discrete recombination line components from 448 pointings. This more than doubled the number of known H II regions in this part of the Galaxy. Continuing the GBT HRDS, the Arecibo HRDS (Bania et al. 2012) discovered 37 previously unknown $H$ II regions in the area $31^{\circ}<\ell<66^{\circ},|b|<1^{\circ}$. Recently, the GBT HRDS has been extended by Anderson et al. (2015), resulting in a further $302 \mathrm{H} \mathrm{II}$ region discoveries. Together, these three northern HRDS surveys achieve a detection rate $>90 \%$, resulting in the discovery of nearly $800 \mathrm{H}$ II regions_-including the most distant Galactic H II regions known.

The Southern H II Region Discovery Survey (SHRDS) is a multi-year project using the Australia Telescope Compact Array (ATCA) to complement the GBT and Arecibo HRDS by extending the survey area into the southern sky $\left(\delta<-45^{\circ}\right)$. This area includes the Southern end of the Galactic Bar, the Near and Far $3 \mathrm{kpc}$ Arms, the Norma/Cygnus Arm, the Scutum/Crux Arm, the Sagitttarius/Carina Arm, and outside the solar circle, the Perseus Arm, and the Outer Arm. Our lists of confirmed $\mathrm{H}$ II regions are seriously incomplete in the third and fourth Galactic quadrants, where no large-scale $\mathrm{H}$ II region RRL survey has been done in nearly three decades (since the work of Caswell \& Haynes 1987). Currently, three candidate $\mathrm{H}$ II regions exist for every confirmed $\mathrm{H}$ II region between $225^{\circ}<\ell<340^{\circ}$, i.e., outside the declination range observable with the GBT.

We make use of the ATCA's compact array configurations, wide band $\mathrm{C} / \mathrm{X}$ receiver and Compact Array Broadband 
Table 1

Pilot SHRDS Source Candidates

\begin{tabular}{|c|c|c|c|c|c|}
\hline $\begin{array}{l}\text { Source } \\
\text { Name }\end{array}$ & $\begin{array}{l}\text { Obs. } \\
\text { Epoch }\end{array}$ & $\begin{array}{l}\# u v \\
\text { Cuts }\end{array}$ & $\begin{array}{c}\text { Int. } \\
\text { (minutes) }\end{array}$ & IRAS Source & $\begin{array}{c}\text { Known } \\
\text { Velocities }\end{array}$ \\
\hline \multicolumn{6}{|c|}{ H II Region Candidates } \\
\hline G214.250-02.461 & 2 & 6 & 25 & IRAS 06425-0214 & $\cdots$ \\
\hline G217.497-00.008 & 2 & 6 & 25 & IRAS 06571-0359 & $\ldots$ \\
\hline G217.640-00.057 & 2 & 6 & 25 & IRAS 06573-0408 & $\cdots$ \\
\hline G222.096-01.981 & 2 & 6 & 25 & IRAS 06587-0859 & $\mathrm{Y}$ \\
\hline G222.159-02.163 & 2 & 6 & 25 & $\cdots$ & $\mathrm{Y}$ \\
\hline G230.354-00.597 & 2 & 7 & 28 & IRAS 07195-1538 & $\cdots$ \\
\hline G234.267-01.496 & 2 & 7 & 28 & IRAS 07240-1930 & $\cdots$ \\
\hline G234.673-00.243 & 2 & 7 & 28 & IRAS 07295-1915 & $\mathrm{Y}$ \\
\hline G234.762-00.277 & 2 & 7 & 28 & IRAS 07296-1921 & $\mathrm{Y}$ \\
\hline G235.696-01.243 & 2 & 7 & 28 & IRAS 07279-2038 & $\mathrm{Y}$ \\
\hline G237.232-01.066 & 2 & 7 & 28 & IRAS 07318-2153 & $\mathrm{Y}$ \\
\hline G237.257-01.281 & 2 & 7 & 28 & IRAS 07310-2201 & $\mathrm{Y}$ \\
\hline G239.332-02.738 & 2 & 7 & 28 & IRAS 07299-2432 & $\mathrm{Y}$ \\
\hline G290.012-00.867 & 2 & 7 & 25 & IRAS 10595-6041 & $\cdots$ \\
\hline G290.385-01.042 & 2 & 6 & 25 & $\cdots$ & $\cdots$ \\
\hline G290.674-00.133 & 2 & 6 & 25 & IRAS 11069-6016 & $\cdots$ \\
\hline G291.596-00.239 & 2 & 6 & 25 & IRAS 11137-0239 & $\cdots$ \\
\hline $\mathrm{G} 292.722+00.157$ & 2 & 7 & 25 & IRAS $11233-6043$ & $\ldots$ \\
\hline G292.889-00.831 & 2 & 7 & 25 & IRAS $11220-6142$ & $\cdots$ \\
\hline G293.483-00.903 & 2 & 5 & 25 & IRAS $11265-6158$ & $\mathrm{Y}$ \\
\hline G293.936-00.873 & 2 & 6 & 25 & $\cdots$ & $\mathrm{Y}$ \\
\hline G293.994-00.934 & 2 & 6 & 25 & $\cdots$ & $\mathrm{Y}$ \\
\hline G294.656-00.438 & 2 & 13 & 52 & $\cdots$ & $\cdots$ \\
\hline G294.988-00.538 & 2 & 12 & 48 & IRAS 11396-6202 & $\mathrm{Y}$ \\
\hline G295.275-00.255 & 2 & 7 & 25 & IRAS 11427-6151 & $\cdots$ \\
\hline G297.248-00.754 & 2 & 7 & 28 & IRAS $11583-6247$ & $\cdots$ \\
\hline G297.626-00.906 & 2 & 7 & 28 & IRAS $12013-6300$ & $\ldots$ \\
\hline $\mathrm{G} 298.473+00.104$ & 2 & 7 & 28 & $\ldots$ & $\cdots$ \\
\hline G298.669+00.064 & 2 & 7 & 28 & IRAS $12117-6213$ & $\cdots$ \\
\hline G299.505+00.025 & 2 & 7 & 28 & $\cdots$ & $\cdots$ \\
\hline G300.519-00.409 & 2 & 7 & 28 & IRAS $12271-6253$ & $\cdots$ \\
\hline G300.972+00.994 & 2 & 7 & 29 & IRAS $12321-6132$ & $\cdots$ \\
\hline G300.983+01.117 & 2 & 7 & 28 & IRAS $12320-6122$ & $\mathrm{Y}$ \\
\hline G313.671-00.105 & 1 & 5 & 51 & IRAS 14183-6050 & $\mathrm{Y}$ \\
\hline $\mathrm{G} 314.219+00.344$ & 1 & 4 & 14 & $\cdots$ & $\mathrm{Y}$ \\
\hline G316.516-00.600 & 1 & 4 & 40 & IRAS $14412-6013$ & $\mathrm{Y}$ \\
\hline $\mathrm{G} 317.861+00.160$ & 1 & 3 & 10 & IRAS $14482-5857$ & $\mathrm{Y}$ \\
\hline $\mathrm{G} 318.248+00.151$ & 1 & 3 & 15 & $\cdots$ & $\cdots$ \\
\hline G319.229+00.225 & 1 & 3 & 31 & $\ldots$ & $\cdots$ \\
\hline G323.449+00.095 & 1 & 4 & 22 & IRAS $15246-5612$ & $\mathrm{Y}$ \\
\hline G323.743-00.249 & 1 & 5 & 31 & IRAS $15278-5620$ & $\mathrm{Y}$ \\
\hline $\mathrm{G} 323.806+00.020$ & 1 & 4 & 16 & IRAS $15270-5604$ & $\cdots$ \\
\hline G323.936-00.037 & 1 & 3 & 19 & $\cdots$ & $\cdots$ \\
\hline G324.662-00.331 & 1 & 3 & 10 & IRAS $15335-5552$ & $\cdots$ \\
\hline G325.108+00.054 & 1 & 3 & 22 & IRAS $15347-5518$ & $\ldots$ \\
\hline G325.354+00.035 & 1 & 4 & 16 & IRAS 15392-5545 & $\cdots$ \\
\hline $\mathrm{G} 326.721+00.773$ & 1 & 3 & 10 & IRAS 15404-5345 & $\mathrm{Y}$ \\
\hline G326.890-00.277 & 1 & 3 & 10 & IRAS $15457-5429$ & $\mathrm{Y}$ \\
\hline G326.916-01.100 & 1 & 3 & 10 & IRAS 15495-5505 & $\mathrm{Y}$ \\
\hline $\mathrm{G} 327.401+00.484$ & 1 & 3 & 10 & IRAS 15454-5335 & $\mathrm{Y}$ \\
\hline G327.555-00.829 & 1 & 3 & 10 & $\cdots$ & $\mathrm{Y}$ \\
\hline G327.714+00.576 & 1 & 3 & 31 & $\cdots$ & $\cdots$ \\
\hline G327.763-00.163 & 1 & 3 & 17 & $\cdots$ & $\cdots$ \\
\hline \multicolumn{6}{|c|}{ Known H II Regions } \\
\hline $\mathrm{G} 213.833+00.618$ & 2 & 6 & 25 & IRAS 06527-0027 & $\mathrm{Y}$ \\
\hline G290.323-02.984 & 2 & 7 & 25 & IRAS $10545-6244$ & $\mathrm{Y}$ \\
\hline G295.748-00.207 & 2 & 7 & 28 & IRAS $11467-6155$ & $\mathrm{Y}$ \\
\hline G315.312-00.273 & 1 & 4 & 14 & $\cdots$ & $\mathrm{Y}$ \\
\hline G313.790+00.706 & 1 & 5 & 14 & IRAS $14170-6002$ & $\mathrm{Y}$ \\
\hline G323.464-00.079 & 1 & 4 & 40 & IRAS $15254-5621$ & $\mathrm{Y}$ \\
\hline G327.313-00.536 & 1 & 3 & 11 & IRAS 15492-5426 & $\mathrm{Y}$ \\
\hline
\end{tabular}

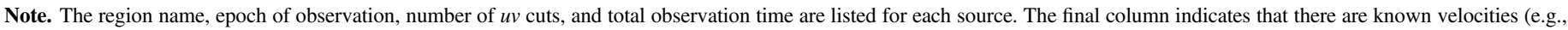
stellar or molecular) probably associated with the region. 
Backend (CABB, Wilson et al. 2011) to observe up to $25 \alpha$ RRL transitions simultaneously. The transitions and polarizations can be averaged together in order to produce a single average $\langle\mathrm{Hn} \alpha\rangle$ spectrum, providing roughly a factor of five improvement in sensitivity compared with a conventional single line observation. With a moderate integration time per candidate, the ATCA can improve the detection threshold of an RRL survey by nearly an order of magnitude compared to the Caswell \& Haynes (1987) Parkes catalog.

The analysis of the pilot survey data was done entirely on the $u v$ plane. The sparse $u v$ coverage for each candidate is not sufficient to make images with good dynamic range. The full SHRDS survey will collect data with multiple telescope configurations and many hour-angle scans on each source, so that maps of the continuum and spectral line emission with good resolution and fidelity can be obtained.

This paper presents the results of two SHRDS pilot observing sessions, in 2013 and 2014, and introduces the telescope and receiver configuration used for the SHRDS. The Pilot Survey source selection, observation, and data reduction strategies are discussed in Sections 2-4. The results of the pilot observations are presented in Table 3.

\section{Source Selection}

The HRDS and SHRDS are not blind surveys complete over defined areas; instead, the survey observations are targeted toward $\mathrm{H}$ II region candidates. Candidate selection is based on infrared and radio continuum morphology, as discussed by Anderson et al. (2014). In the third and fourth Galactic quadrants, the mid-infrared data comes from the WISE catalog of Galactic H II regions, which contains roughly 2000 radioloud candidates. In addition to the WISE (Wright et al. 2010) data at 12 and $22 \mu \mathrm{m}$ wavelength, we also consider Spitzer GLIMPSE at $8 \mu \mathrm{m}$ (Benjamin et al. 2003; Churchwell et al. 2009) and Spitzer MIPSGAL at $24 \mu \mathrm{m}$ (Carey et al. 2009). The radio continuum data comes primarily from the SUMSS survey (Bock et al. 1999), with reference also to the MAGPIS, NVSS, and SGPS surveys (Condon et al. 1998; McClure-Griffiths et al. 2005; Helfand et al. 2006, respectively). To predict the flux density at $\lambda 6 \mathrm{~cm}$, we assumed an optically thin spectral index of $\alpha=-0.12$. The list of targets for the pilot observations is given in Table 1 .

\subsection{Pilot Survey Source Selection}

The SHRDS pilot observations were done in two sessions. Epoch I, observed 2013 June 30, focused on candidates that were expected to show bright RRL detections, which they did. Epoch II, observed 2014 June 26 and 27, used a list of candidates with expected flux densities typical of the SHRDS catalog as a whole. The two epochs also used different longitude ranges in order to generate samples of $\mathrm{H}$ II regions with different Galactic radii.

\subsubsection{Epoch I}

For the first round of observations, we observed $\mathrm{H}$ II region candidates in the range $312^{\circ}<\ell<328^{\circ}$. This section of the fourth Galactic quadrant provides lines of sight that are near the tangents to the Norma-Cygus and Scutum-Crux arms in the inner Galaxy, and are roughly perpendicular to the ScutumCentaurus and Sagittarius Arm tangents in the outer Galaxy.
We selected twenty $\mathrm{H}$ II region candidates (from Anderson et al. 2014) for observations in Epoch I. In addition, four known H II regions: G315.312-00.272 and G327.300-00.548 (from Caswell \& Haynes 1987), and G313.790+00.706 and G323.464-00.079 (from Misanovic et al. 2002), were included in the observation schedule. Thus, Epoch I included a total of 24 targets (Table 1). RRLs from all candidates and known regions were detected.

\subsubsection{Epoch II}

The observations in Epoch II covered longitude range $213^{\circ}<\ell<301^{\circ}$. This area selects mostly $\mathrm{H}$ II regions with Galactocentric radii outside the solar circle. This part of the disk has been little studied in previous Galactic RRL surveys. Between $213^{\circ}<\ell<301^{\circ}$ the ratio of known:candidate $\mathrm{H}$ II regions is $2: 5$, compared with $1: 1$ in the corresponding longitude range in the first and second quadrants $\left(59^{\circ}<\ell<147^{\circ}\right)$ for candidates selected according to the same criteria. Overall, the detection rate for the $\mathrm{H}$ II region candidates observed in Epoch II was $48 \%$ (16 out of 33). Of the 13 candidates in the third quadrant, only 1 was detected, G230.354-00.597, plus the known source G213.833+00.618. In both cases, the lines are only just above the $3 \sigma$ threshold.

Epoch II candidates were selected to have WISE radii between $60^{\prime \prime}$ and $150^{\prime \prime}$ and no known radial velocity information as tabulated by Anderson et al. (2014). We selected $33 \mathrm{H}$ II region candidates between $213^{\circ}<\ell<240^{\circ}$ and $290^{\circ}<\ell<301^{\circ}$ that fulfilled these criteria. Therefore, Epoch II observed a more representative sample from the catalog of Anderson et al. (2014) to determine detection statistics for the SHRDS full survey.

As in Epoch I, a few known H II regions were added to the observation schedule: G213.883+00.618, G290.323-02.984, and G295.748-00.207. Of these three, G295.748-00.207 was strongly detected, and G213.883+00.618 and G290.323 -02.984 were detected at the $3.5 \sigma$ and $5.6 \sigma$ levels, respectively.

\section{Observations}

All pilot SHRDS observations used the ATCA in the five antenna $\mathrm{H} 75$ array configuration, giving a nominal maximum baseline of $75 \mathrm{~m}$ and a beam size of $\mathrm{FWHM} \simeq 65^{\prime \prime}$ at $7.8 \mathrm{GHz}$ depending on the declination and hour angles of the observations. As an interferometer survey, the SHRDS cannot detect emission spread smoothly over much larger angles than the shortest projected baseline, which can be as short as the dish diameter, $22 \mathrm{~m}$. This largest angular scale is roughly equal to the primary beam size, FWHM $=6^{\prime}$ at $7.8 \mathrm{GHz}$. Although the resolution is very coarse, the $\mathrm{H} 75$ configuration gives the best brightness temperature sensitivity, which is the critical parameter for detecting weak spectral line emission from extended sources like most H II regions. Surveys of compact and ultra-compact $\mathrm{HII}$ regions, like the CORNISH and SCORPIO surveys (Hoare et al. 2012; Purcell et al. 2013; Umana et al. 2015) use very different telescope configurations.

The ATCA's CABB (Wilson et al. 2011) and C/X upgrade allow for two $2 \mathrm{GHz}$ spectral windows to be placed anywhere between 4.0 and $10.8 \mathrm{GHz}$. The 64M-32k observing mode used here provides for each of these two windows a coarse resolution spectrum of $32 \times 64 \mathrm{MHz}$ channels and up to 16 


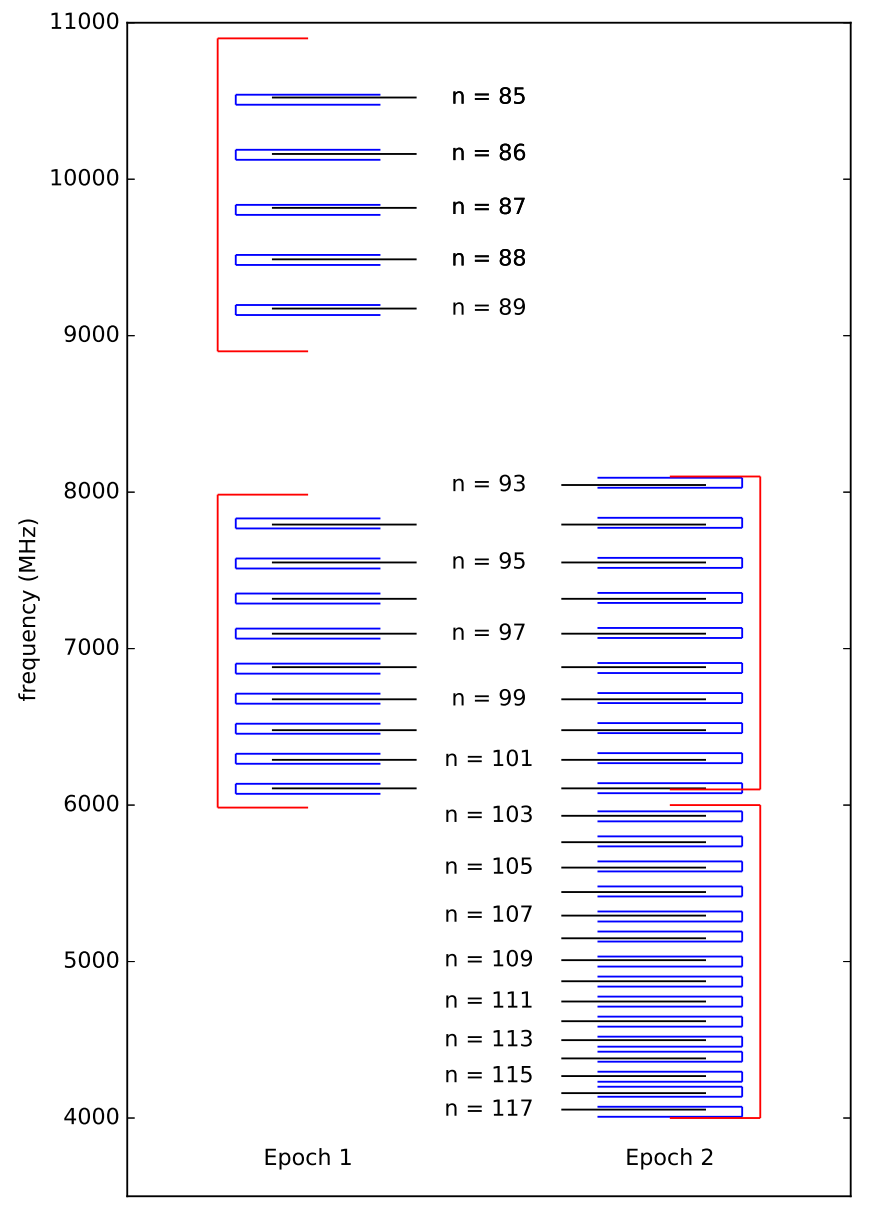

Figure 1. A schematic of CABB frequencies for Epoch I (left) and Epoch II (right). The two $2 \mathrm{GHz} \mathrm{CABB}$ bands are shown in red, the individual zoom bands are shown in blue. The frequencies of the $\mathrm{Hn} \alpha$ recombination lines are shown as black horizontal lines, with the level $n$ indicated.

fine resolution "zoom" bands of 2048 channels across $64 \mathrm{MHz}$, placed within each broadband $2 \mathrm{GHz}$ window. The zoom bands provide very high spectral resolution, with channel separation $32 \mathrm{KHz}=1.2 \mathrm{~km} \mathrm{~s}^{-1}$ at $7.8 \mathrm{GHz}$ and velocity range of nearly $2500 \mathrm{~km} \mathrm{~s}^{-1}$ each. Thus, it is not necessary to center the line rest frequency in each zoom band. The center frequencies of the zoom bands are constrained to have frequency separations equal to integral multiples of half the zoom bandwidth. In practice, selection of zoom band center frequencies is facilitated by the CABB scheduler, part of the ATCA observation scheduling tool (http://www.narrabri.atnf.csiro. au/observing/sched/cabb_nopw/). After calibration and Doppler correction, the zoom bands can be aligned in LSR velocity and resampled to the same channel spacing and then averaged in order to improve the signal-to-noise ratio $(\mathrm{S} / \mathrm{N})$ for weak and marginal detections.

There are $33 \mathrm{Hn} \alpha \mathrm{RRL}$ transitions within the frequency range of the $4 \mathrm{~cm}$ receiver. However, the $\mathrm{H} 86 \alpha$ line is spectrally compromised by higher order RRL transitions (Balser 2006), and the $\mathrm{H} 90 \alpha$ transition can be affected by a trapped mode in the ATCA's $6 / 3 \mathrm{~cm}$ horn. This leaves 31 individual $\mathrm{Hn} \alpha$ transitions between 4.0 and $10.8 \mathrm{GHz}$. The placement of the two $2 \mathrm{GHz} \mathrm{CABB}$ bands, and, hence, the selection of which RRL transitions to observe, is complicated by interference at many frequencies, by variations in the system
Table 2

Recombination Lines Observed

\begin{tabular}{|c|c|c|c|}
\hline $\begin{array}{l}\text { Hn } \alpha \\
n=\end{array}$ & $\begin{array}{l}\nu \text { Rest } \\
\mathrm{MHz}\end{array}$ & $\begin{array}{c}\nu \text { Central } \\
\mathrm{MHz}\end{array}$ & $\underset{\mathrm{km} \mathrm{s}^{-1}}{\Delta V}$ \\
\hline \multicolumn{4}{|c|}{ Epoch I, $\nu_{1}=6984, \nu_{2}=9900 \mathrm{MHz}$} \\
\hline 85 & 10522.04 & 10,508 & 0.9 \\
\hline 86 & 10161.30 & 10,156 & 0.9 \\
\hline 87 & 9816.864 & 9804 & 1.0 \\
\hline 88 & 9487.821 & 9484 & 1.0 \\
\hline 89 & 9173.321 & 9164 & 1.0 \\
\hline 94 & 7792.871 & 7800 & 1.2 \\
\hline 95 & 7550.614 & 7544 & 1.2 \\
\hline 96 & 7318.296 & 7320 & 1.3 \\
\hline 97 & 7095.411 & 7096 & 1.3 \\
\hline 98 & 6881.486 & 6872 & 1.4 \\
\hline 99 & 6676.076 & 6680 & 1.4 \\
\hline 100 & 6478.760 & 6488 & 1.4 \\
\hline 101 & 6289.144 & 6296 & 1.5 \\
\hline 102 & 6106.855 & 6104 & 1.5 \\
\hline \multicolumn{4}{|c|}{ Epoch II, $\nu_{1}=5000, \nu_{2}=7100 \mathrm{MHz}$} \\
\hline 93 & 8045.603 & 8060 & 1.2 \\
\hline 94 & 7792.871 & 7804 & 1.2 \\
\hline 95 & 7550.614 & 7548 & 1.2 \\
\hline 96 & 7318.296 & 7324 & 1.3 \\
\hline 97 & 7095.411 & 7100 & 1.3 \\
\hline 98 & 6881.486 & 6876 & 1.4 \\
\hline 99 & 6676.076 & 6684 & 1.4 \\
\hline 100 & 6478.760 & 6492 & 1.4 \\
\hline 101 & 6289.144 & 6300 & 1.5 \\
\hline 102 & 6106.855 & 6108 & 1.5 \\
\hline 103 & 5931.544 & 5928 & 1.6 \\
\hline 104 & 5762.880 & 5768 & 1.6 \\
\hline 105 & 5600.550 & 5608 & 1.7 \\
\hline 106 & 5444.260 & 5448 & 1.7 \\
\hline 107 & 5293.732 & 5288 & 1.8 \\
\hline 108 & 5148.703 & 5160 & 1.8 \\
\hline 109 & 5008.923 & 5000 & 1.9 \\
\hline 110 & 4874.157 & 4872 & 1.9 \\
\hline 111 & 4744.776 & 4744 & 2.0 \\
\hline 112 & 4618.789 & 4616 & 2.0 \\
\hline 113 & 4497.776 & 4488 & 2.1 \\
\hline 114 & 4380.954 & 4392 & 2.1 \\
\hline 115 & 4268.142 & 4264 & 2.2 \\
\hline 116 & 4159.171 & 4168 & 2.2 \\
\hline 117 & 4053.878 & 4040 & 2.3 \\
\hline
\end{tabular}

Note. $\operatorname{Hn} \alpha$ transitions and rest frequencies are given, with the center frequency and velocity resolution of each zoom band. The center frequencies of the $2 \mathrm{GHz}$ bands $\left(\nu_{1}\right.$ and $\left.\nu_{2}\right)$ are also listed. See Figure 1.

temperature of the receiver with frequency, and by the natural decrease of the line-to-continuum ratio with increasing quantum level. The two epochs of pilot observations explored two among many possible choices of $\mathrm{CABB}$ frequencies (see Figure 1 and Table 2).

Epoch I (project C2842) was observed over 12 hours on 2013 July 1 . The frequency placement of the various bands for Epoch I was chosen to emulate the GBT HRDS observations. Centering the $2 \mathrm{GHz} \mathrm{CABB}$ bands at 7000 and $9900 \mathrm{MHz}$, allows thirteen $\operatorname{Hn} \alpha$ RRLs to be observed simultaneously. This frequency range includes 3 of the RRLs observed by the GBT HRDS and an additional $10 \mathrm{Hn} \alpha$ transitions (see Figure 1). A 
Table 3

Detected RRL Gaussian Parameters

\begin{tabular}{|c|c|c|c|c|c|c|c|c|}
\hline $\begin{array}{l}\text { Source } \\
\text { Name }\end{array}$ & $\begin{array}{l}\operatorname{Hn} \alpha \\
(n)\end{array}$ & $\begin{array}{c}\text { VLSR } \\
\left(\mathrm{km} \mathrm{s}^{-1}\right)\end{array}$ & $\begin{array}{l}\text { FWHM } \\
\left(\mathrm{km} \mathrm{s}^{-1}\right)\end{array}$ & $\begin{array}{l}S_{c} \\
\text { (mJy) }\end{array}$ & $\begin{array}{l}\mathrm{rms} \\
(\mathrm{mJy})\end{array}$ & $\begin{array}{c}S_{L} \\
(\mathrm{mJy})\end{array}$ & $\begin{array}{c}T_{e} \\
(\mathrm{~K})\end{array}$ & $\begin{array}{c}\mathrm{S} / \mathrm{N} \\
\sigma\end{array}$ \\
\hline $\mathrm{G} 213.833+00.618$ & $\mathrm{~S}$ & $53.2 \pm 20.2$ & $33.5 \pm 20.9$ & $\mathrm{~N} / \mathrm{A}$ & 1.4 & $1.9 \pm 2.3$ & $\mathrm{~N} / \mathrm{A}$ & 3.5 \\
\hline G230.354-00.597 & $\mathrm{S}$ & $69.0 \pm 16.1$ & $35.0 \pm 16.7$ & $\mathrm{~N} / \mathrm{A}$ & 1.5 & $2.1 \pm 2.0$ & $\mathrm{~N} / \mathrm{A}$ & 3.7 \\
\hline G290.012-00.867 & $\mathrm{S}$ & $14.7 \pm 8.3$ & $24.9 \pm 8.6$ & $\mathrm{~N} / \mathrm{A}$ & 1.8 & $3.6 \pm 2.5$ & $\mathrm{~N} / \mathrm{A}$ & 4.5 \\
\hline G290.323-02.984 & $\mathrm{S}$ & $-17.7 \pm 8.0$ & $28.3 \pm 8.3$ & $\mathrm{~N} / \mathrm{A}$ & 1.5 & $3.5 \pm 2.1$ & $\mathrm{~N} / \mathrm{A}$ & 5.6 \\
\hline G290.385-01.042 & $\mathrm{S}$ & $9.91 \pm 4.6$ & $13.2 \pm 4.7$ & $\mathrm{~N} / \mathrm{A}$ & 1.9 & $4.0 \pm 2.9$ & $\mathrm{~N} / \mathrm{A}$ & 3.4 \\
\hline G290.674-00.133 & $\mathrm{S}$ & $19.3 \pm 5.2$ & $23.2 \pm 5.3$ & $\mathrm{~N} / \mathrm{A}$ & 1.4 & $5.2 \pm 2.4$ & $\mathrm{~N} / \mathrm{A}$ & 8.0 \\
\hline G291.596-00.239 & $\mathrm{S}$ & $11.4 \pm 5.1$ & $44.2 \pm 5.4$ & $\mathrm{~N} / \mathrm{A}$ & 2.5 & $15.3 \pm 3.7$ & $8300 \pm 1200$ & 18. \\
\hline G292.889-00.831 & $\mathrm{S}$ & $21.8 \pm 7.1$ & $30.0 \pm 7.3$ & $\mathrm{~N} / \mathrm{A}$ & 1.4 & $4.8 \pm 2.3$ & $\mathrm{~N} / \mathrm{A}$ & 8.4 \\
\hline G293.936-00.873 & $\mathrm{S}$ & $36.6 \pm 1.4$ & $22.3 \pm 1.5$ & $\mathrm{~N} / \mathrm{A}$ & 2.0 & $16.4 \pm 2.2$ & $9400 \pm 400$ & 17 \\
\hline G293.994-00.934 & $\mathrm{S}$ & $46.5 \pm 1.7$ & $25.6 \pm 1.8$ & $\mathrm{~N} / \mathrm{A}$ & 1.3 & $18.6 \pm 2.6$ & $9600 \pm 400$ & 32 \\
\hline G294.988-00.538 & $\mathrm{S}$ & $39.8 \pm 1.9$ & $27.4 \pm 2.0$ & $\mathrm{~N} / \mathrm{A}$ & 1.2 & $9.7 \pm 1.4$ & $10000 \pm 400$ & 19 \\
\hline G295.275-00.255 & $\mathrm{S}$ & $30.1 \pm 6.2$ & $30.6 \pm 6.3$ & $\mathrm{~N} / \mathrm{A}$ & 1.6 & $5.6 \pm 2.3$ & $7900 \pm 4500$ & 8.7 \\
\hline G295.748-00.207 & $\mathrm{S}$ & $23.3 \pm 3.5$ & $23.9 \pm 3.5$ & $\mathrm{~N} / \mathrm{A}$ & 1.6 & $7.2 \pm 2.1$ & $7300 \pm 1800$ & 9.8 \\
\hline \multirow[t]{6}{*}{ G297.248-00.754 } & $\mathrm{S}$ & $22.6 \pm 1.5$ & $24.4 \pm 1.6$ & $\mathrm{~N} / \mathrm{A}$ & 1.9 & $22.6 \pm 2.9$ & $7200 \pm 400$ & 26 \\
\hline & 107 & $24.4 \pm 2.7$ & $23.1 \pm 2.8$ & 369 & 7.2 & $22.0 \pm 5.0$ & $7700 \pm 3200$ & 3.1 \\
\hline & 106 & $22.0 \pm 2.8$ & $27.6 \pm 2.8$ & 365 & 7.0 & $21.0 \pm 4.0$ & $6900 \pm 2300$ & 3.0 \\
\hline & 105 & $22.3 \pm 2.6$ & $25.8 \pm 2.7$ & 359 & 6.9 & $20.0 \pm 4.0$ & $7700 \pm 2700$ & 3.0 \\
\hline & 104 & $20.4 \pm 2.3$ & $26.0 \pm 2.3$ & 356 & 8.3 & $25.0 \pm 4.0$ & $6500 \pm 1900$ & 3.1 \\
\hline & 103 & $22.3 \pm 2.8$ & $25.3 \pm 2.9$ & 350 & 7.0 & $21.0 \pm 4.0$ & $7700 \pm 3000$ & 3.1 \\
\hline G297.626-00.906 & $\mathrm{S}$ & $31.3 \pm 2.1$ & $28.4 \pm 2.2$ & $\mathrm{~N} / \mathrm{A}$ & 1.5 & $16.1 \pm 2.5$ & $7900 \pm 400$ & 26 \\
\hline $\mathrm{G} 298.473+00.104$ & $\mathrm{~S}$ & $32.9 \pm 2.0$ & $23.5 \pm 2.1$ & $\mathrm{~N} / \mathrm{A}$ & 2.0 & $12.2 \pm 2.2$ & $7900 \pm 400$ & 13 \\
\hline G298.669+00.064 & $\mathrm{S}$ & $24.1 \pm 2.4$ & $14.1 \pm 2.4$ & $\mathrm{~N} / \mathrm{A}$ & 2.2 & $11.4 \pm 3.9$ & $11000 \pm 3800$ & 8.7 \\
\hline G300.972+00.994 & $\mathrm{S}$ & $-34.5 \pm 7.2$ & $39.1 \pm 7.5$ & $\mathrm{~N} / \mathrm{A}$ & 1.7 & $3.8 \pm 1.4$ & $5600 \pm 2600$ & 6.3 \\
\hline \multirow[t]{9}{*}{ G300.983+01.117 } & $\mathrm{S}$ & $-42.0 \pm 0.4$ & $26.5 \pm 0.4$ & $\mathrm{~N} / \mathrm{A}$ & 3.3 & $83.8 \pm 2.8$ & $6000 \pm 880$ & 26.0 \\
\hline & 107 & $-42.4 \pm 0.8$ & $25.6 \pm 0.9$ & 1360 & 12.3 & $86.0 \pm 5.0$ & $6700 \pm 650$ & 7.0 \\
\hline & 106 & $-43.0 \pm 0.9$ & $25.2 \pm 0.9$ & 1330 & 12.6 & $86.0 \pm 6.0$ & $6800 \pm 690$ & 6.8 \\
\hline & 105 & $-42.7 \pm 0.8$ & $27.2 \pm 0.8$ & 1290 & 13.2 & $91.0 \pm 5.0$ & $6100 \pm 530$ & 6.9 \\
\hline & 104 & $-41.9 \pm 1.0$ & $26.2 \pm 1.0$ & 1260 & 13.3 & $83.0 \pm 6.0$ & $6900 \pm 780$ & 6.3 \\
\hline & 103 & $-42.0 \pm 1.0$ & $25.7 \pm 1.0$ & 1220 & 12.6 & $85.0 \pm 6.0$ & $6800 \pm 770$ & 6.8 \\
\hline & 96 & $-42.2 \pm 1.0$ & $26.0 \pm 1.0$ & 879 & 22.5 & $81.0 \pm 6.0$ & $6500 \pm 720$ & 3.6 \\
\hline & 94 & $-41.2 \pm 1.0$ & $24.9 \pm 1.0$ & 809 & 20.0 & $75.0 \pm 6.0$ & $7100 \pm 850$ & 3.8 \\
\hline & 93 & $-40.6 \pm 1.1$ & $26.6 \pm 1.1$ & 750 & 17.7 & $73.0 \pm 6.0$ & $6600 \pm 810$ & 4.2 \\
\hline G313.671-00.104 & $\mathrm{S}$ & $-54.6 \pm 1.4$ & $24.6 \pm 1.5$ & $\mathrm{~N} / \mathrm{A}$ & 1.6 & $12.0 \pm 1.4$ & $6700 \pm 400$ & 17 \\
\hline \multirow[t]{9}{*}{ G313.790+00.706 } & $\mathrm{S}$ & $-57.2 \pm 0.9$ & $22.6 \pm 0.9$ & $\mathrm{~N} / \mathrm{A}$ & 2.6 & $38.7 \pm 3.0$ & $7000 \pm 450$ & 32 \\
\hline & 101 & $-57.6 \pm 1.9$ & $20.2 \pm 1.9$ & 436 & 11.1 & $41.0 \pm 8.0$ & $6800 \pm 2200$ & 3.7 \\
\hline & 97 & $-56.7 \pm 1.8$ & $20.6 \pm 1.8$ & 399 & 11.2 & $38.0 \pm 6.0$ & $7300 \pm 2100$ & 3.5 \\
\hline & 96 & $-56.8 \pm 2.6$ & $26.9 \pm 2.6$ & 396 & 11.8 & $35.0 \pm 6.0$ & $6400 \pm 2100$ & 3.0 \\
\hline & 95 & $-55.0 \pm 2.2$ & $26.4 \pm 2.3$ & 386 & 11.7 & $35.0 \pm 6.0$ & $6600 \pm 1800$ & 3.1 \\
\hline & 94 & $-58.5 \pm 1.6$ & $20.7 \pm 1.6$ & 372 & 12.8 & $41.0 \pm 6.0$ & $7100 \pm 1700$ & 3.2 \\
\hline & 89 & $-55.7 \pm 1.7$ & $21.7 \pm 1.8$ & 330 & 11.9 & $39.0 \pm 6.0$ & $7400 \pm 1900$ & 3.3 \\
\hline & 87 & $-57.0 \pm 1.8$ & $21.3 \pm 1.9$ & 306 & 11.9 & $40.0 \pm 7.0$ & $7500 \pm 2200$ & 3.4 \\
\hline & 85 & $-56.2 \pm 1.7$ & $22.2 \pm 1.8$ & 294 & 13.8 & $42.0 \pm 6.0$ & $7100 \pm 1800$ & 3.1 \\
\hline \multirow[t]{9}{*}{ G314.219+00.344 } & $\mathrm{S}$ & $-62.5 \pm 0.4$ & $20.0 \pm 0.5$ & $\mathrm{~N} / \mathrm{A}$ & 3.5 & $66.4 \pm 3.1$ & $6500 \pm 640$ & 38 \\
\hline & 101 & $-63.0 \pm 1.0$ & $21.0 \pm 1.0$ & 706 & 12.8 & $81.0 \pm 7.0$ & $5600 \pm 780$ & 6.3 \\
\hline & 97 & $-62.7 \pm 0.9$ & $18.1 \pm 0.9$ & 534 & 12.3 & $71.0 \pm 7.0$ & $6200 \pm 940$ & 5.8 \\
\hline & 96 & $-61.7 \pm 1.4$ & $20.6 \pm 1.4$ & 495 & 12.2 & $55.0 \pm 7.0$ & $6700 \pm 1400$ & 4.6 \\
\hline & 95 & $-63.1 \pm 1.2$ & $18.4 \pm 1.2$ & 468 & 12.1 & $63.0 \pm 8.0$ & $6500 \pm 1300$ & 5.2 \\
\hline & 94 & $-62.1 \pm 1.2$ & $21.8 \pm 1.2$ & 438 & 12.9 & $59.0 \pm 6.0$ & $5700 \pm 990$ & 4.6 \\
\hline & 89 & $-61.6 \pm 1.2$ & $21.2 \pm 1.2$ & 401 & 12.5 & $57.0 \pm 6.0$ & $6500 \pm 1100$ & 4.6 \\
\hline & 87 & $-62.7 \pm 1.0$ & $18.6 \pm 1.0$ & 389 & 13.0 & $67.0 \pm 7.0$ & $6700 \pm 1100$ & 5.1 \\
\hline & 85 & $-62.5 \pm 1.5$ & $20.1 \pm 1.6$ & 360 & 15.3 & $65.0 \pm 10.0$ & $6400 \pm 1600$ & 4.3 \\
\hline \multirow[t]{4}{*}{ G315.312-00.273 } & $\mathrm{S}$ & $14.2 \pm 1.4$ & $24.0 \pm 1.4$ & $\mathrm{~N} / \mathrm{A}$ & 3.6 & $30.0 \pm 3.6$ & $7700 \pm 400$ & 18 \\
\hline & 101 & $10.7 \pm 2.1$ & $18.1 \pm 2.2$ & 414 & 11.5 & $37.0 \pm 9.0$ & $7800 \pm 3300$ & 3.3 \\
\hline & 97 & $14.1 \pm 2.4$ & $22.4 \pm 2.4$ & 377 & 11.9 & $36.0 \pm 7.0$ & $6900 \pm 2500$ & 3.1 \\
\hline & 85 & $15.2 \pm 4.5$ & $33.4 \pm 4.7$ & 240 & 14.9 & $22.0 \pm 6.0$ & $7400 \pm 3900$ & 1.5 \\
\hline \multirow[t]{6}{*}{ G316.516-00.600 } & $\mathrm{S}$ & $-45.6 \pm 0.9$ & $19.9 \pm 0.9$ & $\mathrm{~N} / \mathrm{A}$ & 1.7 & $19.0 \pm 1.7$ & $5900 \pm 400$ & 22 \\
\hline & 97 & $-46.2 \pm 2.0$ & $18.1 \pm 2.0$ & 142 & 6.1 & $20.0 \pm 4.0$ & $5900 \pm 2300$ & 3.3 \\
\hline & 96 & $-45.4 \pm 2.0$ & $19.4 \pm 2.0$ & 140 & 6.5 & $20.0 \pm 4.0$ & $5500 \pm 2000$ & 3.2 \\
\hline & 94 & $-46.2 \pm 1.8$ & $18.2 \pm 1.9$ & 135 & 6.6 & $21.0 \pm 4.0$ & $5900 \pm 2100$ & 3.2 \\
\hline & 89 & $-42.7 \pm 1.8$ & $19.8 \pm 1.8$ & 115 & 6.7 & $21.0 \pm 3.0$ & $5600 \pm 1700$ & 3.2 \\
\hline & 87 & $-45.8 \pm 1.9$ & $20.3 \pm 1.9$ & 106 & 6.9 & $21.0 \pm 4.0$ & $5300 \pm 1700$ & 3.2 \\
\hline $\mathrm{G} 317.861+00.160$ & $\mathrm{~S}$ & $1.53 \pm 0.9$ & $22.9 \pm 0.9$ & $\mathrm{~N} / \mathrm{A}$ & 2.7 & $55.6 \pm 4.6$ & $7600 \pm 430$ & 44 \\
\hline
\end{tabular}


Table 3

(Continued)

\begin{tabular}{|c|c|c|c|c|c|c|c|c|}
\hline $\begin{array}{l}\text { Source } \\
\text { Name }\end{array}$ & $\begin{array}{l}\operatorname{Hn} \alpha \\
(n)\end{array}$ & $\begin{array}{c}\text { VLSR } \\
\left(\mathrm{km} \mathrm{s}^{-1}\right)\end{array}$ & $\begin{array}{l}\text { FWHM } \\
\left(\mathrm{km} \mathrm{s}^{-1}\right)\end{array}$ & $\begin{array}{l}S_{c} \\
(\mathrm{mJy})\end{array}$ & $\begin{array}{l}\mathrm{rms} \\
(\mathrm{mJy})\end{array}$ & $\begin{array}{c}S_{L} \\
(\mathrm{mJy})\end{array}$ & $\begin{array}{c}T_{e} \\
(\mathrm{~K})\end{array}$ & $\begin{array}{c}\mathrm{S} / \mathrm{N} \\
\sigma\end{array}$ \\
\hline & 101 & $1.68 \pm 1.7$ & $24.4 \pm 1.7$ & 713 & 13.0 & $55.0 \pm 7.0$ & $6900 \pm 1500$ & 4.2 \\
\hline & 97 & $2.22 \pm 1.6$ & $25.0 \pm 1.6$ & 648 & 13.2 & $55.0 \pm 7.0$ & $7000 \pm 1400$ & 4.2 \\
\hline & 96 & $1.49 \pm 1.3$ & $19.6 \pm 1.4$ & 631 & 13.8 & $66.0 \pm 9.0$ & $7400 \pm 1600$ & 4.8 \\
\hline & 95 & $1.24 \pm 1.5$ & $20.7 \pm 1.5$ & 614 & 13.3 & $54.0 \pm 8.0$ & $8400 \pm 2000$ & 4.1 \\
\hline & 94 & $2.88 \pm 1.9$ & $28.5 \pm 2.0$ & 597 & 14.4 & $53.0 \pm 7.0$ & $6600 \pm 1400$ & 3.7 \\
\hline & 89 & $0.942 \pm 1.4$ & $21.3 \pm 1.5$ & 506 & 13.9 & $55.0 \pm 7.0$ & $8200 \pm 1700$ & 4.0 \\
\hline & 87 & $0.801 \pm 1.7$ & $23.8 \pm 1.8$ & 466 & 14.8 & $52.0 \pm 7.0$ & $7800 \pm 1800$ & 3.5 \\
\hline & 85 & $-0.447 \pm 2.4$ & $29.8 \pm 2.5$ & 431 & 16.5 & $49.0 \pm 8.0$ & $6800 \pm 1900$ & 3.0 \\
\hline \multirow[t]{2}{*}{ G318.248+00.151 } & $\mathrm{S}$ & $-39.9 \pm 2.0$ & $19.0 \pm 2.0$ & $\mathrm{~N} / \mathrm{A}$ & 2.8 & $17.0 \pm 3.7$ & $5900 \pm 740$ & 12 \\
\hline & 101 & $-43.0 \pm 1.7$ & $12.2 \pm 1.7$ & 175 & 11.0 & $34.0 \pm 10.0$ & $5500 \pm 3000$ & 3.2 \\
\hline G319.229+00.225 & $\mathrm{S}$ & $-66.1 \pm 1.8$ & $20.9 \pm 1.8$ & $\mathrm{~N} / \mathrm{A}$ & 1.9 & $12.9 \pm 2.2$ & $6800 \pm 400$ & 14 \\
\hline G323.449+00.095 & $\mathrm{S}$ & $-75.1 \pm 1.7$ & $22.5 \pm 1.7$ & $\mathrm{~N} / \mathrm{A}$ & 2.0 & $13.8 \pm 2.1$ & $5900 \pm 400$ & 15 \\
\hline \multirow[t]{11}{*}{ G323.464-00.079 } & $\mathrm{S}$ & $-68.8 \pm 1.2$ & $38.3 \pm 1.2$ & $\mathrm{~N} / \mathrm{A}$ & 1.3 & $28.0 \pm 2.2$ & $11000 \pm 1700$ & 45 \\
\hline & $\mathrm{S} 2$ & $-68.1 \pm 1.2$ & $27.7 \pm 2$ & $\mathrm{~N} / \mathrm{A}$ & 1.3 & $24 \pm 2.2$ & $\ldots$ & 21 \\
\hline & $\mathrm{S} 2$ & $-77.2 \pm 1.2$ & $105.5 \pm 10$ & $\mathrm{~N} / \mathrm{A}$ & 1.3 & $6.5 \pm 2.2$ & $\ldots$ & 4.9 \\
\hline & 101 & $-66.4 \pm 2.5$ & $31.2 \pm 2.6$ & 589 & 6.3 & $20.0 \pm 3.0$ & $11000 \pm 2900$ & 3.2 \\
\hline & 97 & $-67.1 \pm 1.7$ & $27.7 \pm 1.8$ & 636 & 6.2 & $26.0 \pm 3.0$ & $12000 \pm 2300$ & 4.3 \\
\hline & 96 & $-67.1 \pm 1.8$ & $27.3 \pm 1.9$ & 649 & 6.6 & $26.0 \pm 3.0$ & $13000 \pm 2700$ & 4.0 \\
\hline & 95 & $-67.7 \pm 2.0$ & $27.3 \pm 2.1$ & 661 & 6.5 & $24.0 \pm 3.0$ & $14000 \pm 3400$ & 3.8 \\
\hline & 94 & $-69.3 \pm 1.9$ & $30.7 \pm 1.9$ & 673 & 7.1 & $27.0 \pm 3.0$ & $12000 \pm 2200$ & 3.9 \\
\hline & 89 & $-68.5 \pm 1.6$ & $35.2 \pm 1.6$ & 746 & 7.7 & $35.0 \pm 3.0$ & $11000 \pm 1500$ & 4.6 \\
\hline & 87 & $-68.6 \pm 1.3$ & $36.6 \pm 1.3$ & 770 & 8.2 & $40.0 \pm 2.0$ & $11000 \pm 1100$ & 4.9 \\
\hline & 85 & $-68.8 \pm 1.4$ & $36.9 \pm 1.4$ & 804 & 9.2 & $42.0 \pm 3.0$ & $11000 \pm 1200$ & 4.7 \\
\hline \multirow[t]{7}{*}{ G323.743-00.249 } & $\mathrm{S}$ & $-47.3 \pm 0.7$ & $19.2 \pm 0.7$ & $\mathrm{~N} / \mathrm{A}$ & 2.2 & $22.8 \pm 1.8$ & $6000 \pm 400$ & 20 \\
\hline & 101 & $-47.3 \pm 1.9$ & $17.0 \pm 1.9$ & 165 & 6.9 & $22.0 \pm 5.0$ & $5800 \pm 2300$ & 3.2 \\
\hline & 97 & $-46.7 \pm 1.6$ & $17.7 \pm 1.6$ & 160 & 6.8 & $25.0 \pm 4.0$ & $5500 \pm 1700$ & 3.8 \\
\hline & 95 & $-47.7 \pm 2.0$ & $19.2 \pm 2.1$ & 157 & 6.8 & $21.0 \pm 4.0$ & $6100 \pm 2300$ & 3.2 \\
\hline & 89 & $-47.8 \pm 1.4$ & $17.3 \pm 1.4$ & 147 & 7.1 & $27.0 \pm 4.0$ & $6300 \pm 1600$ & 3.8 \\
\hline & 87 & $-47.6 \pm 1.6$ & $21.7 \pm 1.7$ & 141 & 7.3 & $26.0 \pm 4.0$ & $5400 \pm 1400$ & 3.6 \\
\hline & 85 & $-46.1 \pm 2.3$ & $19.4 \pm 2.4$ & 138 & 7.6 & $25.0 \pm 6.0$ & $6600 \pm 3000$ & 3.3 \\
\hline \multirow[t]{5}{*}{ G323.806+00.021 } & $\mathrm{S}$ & $-58.9 \pm 0.9$ & $21.6 \pm 0.9$ & $\mathrm{~N} / \mathrm{A}$ & 2.7 & $31.6 \pm 2.7$ & $7200 \pm 400$ & 24 \\
\hline & 101 & $-57.4 \pm 2.0$ & $18.8 \pm 2.0$ & 373 & 10.0 & $35.0 \pm 7.0$ & $7300 \pm 2700$ & 3.5 \\
\hline & 97 & $-57.0 \pm 1.7$ & $18.4 \pm 1.7$ & 333 & 10.0 & $36.0 \pm 6.0$ & $7400 \pm 2300$ & 3.6 \\
\hline & 96 & $-59.3 \pm 2.1$ & $25.4 \pm 2.1$ & 320 & 10.4 & $37.0 \pm 6.0$ & $5400 \pm 1400$ & 3.6 \\
\hline & 89 & $-59.4 \pm 1.8$ & $21.0 \pm 1.9$ & 248 & 11.0 & $35.0 \pm 6.0$ & $6700 \pm 2000$ & 3.2 \\
\hline G323.936-00.037 & $\mathrm{S}$ & $-57.3 \pm 4.5$ & $24.5 \pm 4.6$ & $\mathrm{~N} / \mathrm{A}$ & 2.4 & $13.8 \pm 5.2$ & $5600 \pm 2600$ & 12.7 \\
\hline G324.662-00.331 & $\mathrm{S}$ & $-47.8 \pm 1.7$ & $21.3 \pm 1.8$ & $\mathrm{~N} / \mathrm{A}$ & 2.9 & $28.7 \pm 4.8$ & $7200 \pm 400$ & 20 \\
\hline $\mathrm{G} 325.108+00.054$ & $\mathrm{~S}$ & $-67.8 \pm 2.7$ & $20.4 \pm 2.7$ & $\mathrm{~N} / \mathrm{A}$ & 3.2 & $11.5 \pm 3.1$ & $6700 \pm 1600$ & 7.3 \\
\hline \multirow[t]{14}{*}{ G325.354-00.035 } & $\mathrm{S}$ & $-63.8 \pm 0.6$ & $34.4 \pm 1.5$ & $\mathrm{~N} / \mathrm{A}$ & 1.4 & $15 \pm 1.5$ & $6800 \pm 800$ & 26 \\
\hline & 102 & $-63.3 \pm 1.4$ & $25.2 \pm 3.4$ & 251 & 4.4 & $17 \pm 2.0$ & $7232 \pm 970$ & 8.7 \\
\hline & 101 & $-68.8 \pm 2.0$ & $31.3 \pm 4.7$ & 252 & 4.6 & $14 \pm 1.9$ & $7168 \pm 1100$ & 7.7 \\
\hline & 100 & $-62.7 \pm 2.0$ & $36.8 \pm 4.8$ & 246 & 5.2 & $17 \pm 2.0$ & $5335 \pm 690$ & 8.9 \\
\hline & 98 & $-59.1 \pm 1.1$ & $19.6 \pm 2.7$ & 235 & 6.1 & $26 \pm 3.1$ & $6630 \pm 920$ & 8.4 \\
\hline & 97 & $-61.4 \pm 2.4$ & $37.9 \pm 6.0$ & 228 & 5.5 & $19 \pm 2.1$ & $4934 \pm 800$ & 9.2 \\
\hline & 96 & $-60.2 \pm 1.3$ & $19.4 \pm 3.4$ & 222 & 6.0 & $24 \pm 3.2$ & $7142 \pm 1300$ & 7.7 \\
\hline & 95 & $-62.5 \pm 2.0$ & $27.4 \pm 4.7$ & 217 & 5.6 & $16 \pm 2.4$ & $7574 \pm 1400$ & 6.7 \\
\hline & 94 & $-65.4 \pm 1.8$ & $32.2 \pm 4.3$ & 211 & 5.0 & $17 \pm 2.0$ & $6244 \pm 830$ & 8.7 \\
\hline & 89 & $-63.8 \pm 2.1$ & $31.3 \pm 5.1$ & 183 & 4.6 & $13 \pm 1.8$ & $8501 \pm 1400$ & 7.1 \\
\hline & 88 & $-60.6 \pm 1.6$ & $28.9 \pm 3.7$ & 178 & 4.6 & $17 \pm 1.9$ & $7130 \pm 910$ & 9.0 \\
\hline & 87 & $-60.9 \pm 1.3$ & $30.1 \pm 3.1$ & 172 & 4.6 & $22 \pm 1.9$ & $5746 \pm 570$ & 11.4 \\
\hline & 86 & $-56.5 \pm 1.8$ & $25.9 \pm 4.3$ & 167 & 5.7 & $18 \pm 2.5$ & $7869 \pm 1350$ & 7.0 \\
\hline & 85 & $-59.8 \pm 1.3$ & $21.7 \pm 3.1$ & 162 & 5.2 & $21 \pm 2.5$ & $8035 \pm 1140$ & 8.2 \\
\hline \multirow[t]{5}{*}{ G326.721+00.773 } & $\mathrm{S}$ & $-40.6 \pm 0.9$ & $22.6 \pm 0.9$ & $\mathrm{~N} / \mathrm{A}$ & 4.9 & $45.5 \pm 3.7$ & $6300 \pm 400$ & 20 \\
\hline & 101 & $-40.9 \pm 2.0$ & $18.8 \pm 2.0$ & 458 & 14.3 & $51.0 \pm 11.0$ & $6300 \pm 2300$ & 3.6 \\
\hline & 97 & $-40.6 \pm 2.0$ & $23.8 \pm 2.0$ & 470 & 15.4 & $53.0 \pm 9.0$ & $5700 \pm 1500$ & 3.5 \\
\hline & 96 & $-40.2 \pm 2.0$ & $25.4 \pm 2.1$ & 480 & 16.4 & $56.0 \pm 9.0$ & $5400 \pm 1400$ & 3.5 \\
\hline & 95 & $-40.3 \pm 2.2$ & $22.0 \pm 2.2$ & 466 & 15.7 & $46.0 \pm 9.0$ & $7200 \pm 2400$ & 3.0 \\
\hline \multirow[t]{5}{*}{ G326.890-00.277 } & $\mathrm{S}$ & $-44.2 \pm 0.7$ & $19.1 \pm 0.7$ & $\mathrm{~N} / \mathrm{A}$ & 3.5 & $63.2 \pm 4.5$ & $6200 \pm 440$ & 35 \\
\hline & 101 & $-45.4 \pm 1.8$ & $19.4 \pm 1.8$ & 487 & 13.0 & $49.0 \pm 9.0$ & $6700 \pm 2000$ & 3.8 \\
\hline & 97 & $-43.7 \pm 1.2$ & $18.1 \pm 1.2$ & 465 & 13.4 & $65.0 \pm 8.0$ & $6000 \pm 1200$ & 4.9 \\
\hline & 96 & $-45.0 \pm 1.8$ & $22.6 \pm 1.9$ & 456 & 13.8 & $53.0 \pm 8.0$ & $5900 \pm 1600$ & 3.9 \\
\hline & 95 & $-45.3 \pm 1.6$ & $22.2 \pm 1.7$ & 450 & 13.6 & $54.0 \pm 8.0$ & $6000 \pm 1400$ & 4.0 \\
\hline
\end{tabular}


Table 3

(Continued)

\begin{tabular}{|c|c|c|c|c|c|c|c|c|}
\hline $\begin{array}{l}\text { Source } \\
\text { Name }\end{array}$ & $\begin{array}{l}\operatorname{Hn} \alpha \\
(n)\end{array}$ & $\begin{array}{c}\text { VLSR } \\
\left(\mathrm{km} \mathrm{s}^{-1}\right)\end{array}$ & $\begin{array}{c}\text { FWHM } \\
\left(\mathrm{km} \mathrm{s}^{-1}\right)\end{array}$ & $\begin{array}{l}S_{c} \\
(\mathrm{mJy})\end{array}$ & $\begin{array}{l}\mathrm{rms} \\
(\mathrm{mJy})\end{array}$ & $\begin{array}{c}S_{L} \\
(\mathrm{mJy})\end{array}$ & $\begin{array}{c}T_{e} \\
(\mathrm{~K})\end{array}$ & $\begin{array}{c}\mathrm{S} / \mathrm{N} \\
\sigma\end{array}$ \\
\hline & 94 & $-43.4 \pm 1.2$ & $18.3 \pm 1.2$ & 445 & 15.7 & $62.0 \pm 8.0$ & $6500 \pm 1300$ & 4.0 \\
\hline & 89 & $-43.7 \pm 0.9$ & $18.7 \pm 0.9$ & 415 & 13.9 & $75.0 \pm 7.0$ & $6000 \pm 880$ & 5.4 \\
\hline & 85 & $-43.7 \pm 0.8$ & $17.9 \pm 0.8$ & 396 & 15.1 & $80.0 \pm 7.0$ & $6400 \pm 900$ & 5.3 \\
\hline G326.916-01.100 & $\mathrm{S}$ & $-49.8 \pm 1.8$ & $21.0 \pm 1.9$ & $\mathrm{~N} / \mathrm{A}$ & 4.4 & $18.9 \pm 3.4$ & $6800 \pm 450$ & 8.8 \\
\hline \multirow[t]{7}{*}{ G327.313-00.536 } & S & $-48.5 \pm 0.2$ & $28.1 \pm 0.3$ & $\mathrm{~N} / \mathrm{A}$ & 33.1 & $2280.0 \pm 41.7$ & $6100 \pm 890$ & 163 \\
\hline & 97 & $-48.7 \pm 0.3$ & $28.0 \pm 0.3$ & 25100 & 239.0 & $2226.0 \pm 46.0$ & $6100 \pm 400$ & 9.3 \\
\hline & 96 & $-48.7 \pm 0.3$ & $28.0 \pm 0.3$ & 24600 & 238.0 & $2293.0 \pm 48.0$ & $6000 \pm 400$ & 9.6 \\
\hline & 95 & $-48.6 \pm 0.3$ & $28.2 \pm 0.3$ & 24100 & 234.0 & $2289.0 \pm 50.0$ & $6100 \pm 400$ & 9.8 \\
\hline & 94 & $-48.5 \pm 0.3$ & $27.6 \pm 0.3$ & 23500 & 303.0 & $2315.0 \pm 53.0$ & $6200 \pm 400$ & 7.6 \\
\hline & 89 & $-48.6 \pm 0.3$ & $28.1 \pm 0.3$ & 20300 & 270.0 & $2420.0 \pm 50.0$ & $6000 \pm 400$ & 9.0 \\
\hline & 87 & $-48.3 \pm 0.3$ & $27.6 \pm 0.3$ & 19100 & 282.0 & $2479.0 \pm 53.0$ & $6100 \pm 400$ & 8.8 \\
\hline G327.714+00.577 & $\mathrm{S}$ & $-47.4 \pm 2.4$ & $21.9 \pm 2.5$ & $\mathrm{~N} / \mathrm{A}$ & 2.1 & $12.0 \pm 2.7$ & $6600 \pm 1000$ & 12 \\
\hline \multirow[t]{4}{*}{ G327.763+00.163 } & $\mathrm{S}$ & $-92.8 \pm 1.2$ & $21.6 \pm 1.2$ & $\mathrm{~N} / \mathrm{A}$ & 2.6 & $30.1 \pm 3.4$ & $6800 \pm 400$ & 24 \\
\hline & 97 & $-93.5 \pm 2.1$ & $19.8 \pm 2.1$ & 297 & 10.3 & $33.0 \pm 7.0$ & $6700 \pm 2500$ & 3.3 \\
\hline & 94 & $-93.5 \pm 1.8$ & $21.2 \pm 1.8$ & 265 & 10.7 & $37.0 \pm 6.0$ & $5700 \pm 1600$ & 3.5 \\
\hline & 87 & $-92.9 \pm 2.0$ & $19.2 \pm 2.0$ & 191 & 10.8 & $35.0 \pm 7.0$ & $6100 \pm 2300$ & 3.3 \\
\hline
\end{tabular}

(This table is available in machine-readable form.)

single phase calibrator, PKS B1421-490, was used for all Epoch I observations. Both epochs used PKS 1934-63 as a flux calibrator, and phase calibration was done every $\sim 20$ minutes. Each source was observed at several-hour angles to improve the sampling on the $u v$ plane.

Epoch II (project C2963) was observed over two 12-hour blocks on 2014 July 26 and 27 . The centers of the two broadband IFs were moved to 5000 and $7100 \mathrm{MHz}$ in order to increase the number of simultaneously observed $\mathrm{Hn} \alpha$ transitions from 13 to 25 . In addition to PKS 1934-638, secondary bandpass calibrators (PKS0823-500 and PKS0537-441) were also observed in Epoch II. As the Epoch II targets fell into two longitude groups $\left(\ell \approx 220^{\circ}\right.$ and $\left.\ell \approx 295^{\circ}\right)$, two phase calibration sources were chosen for each day: PKS0723-008 and PMS J1131-58 for July 26, and PKS0727-115 and PKS1148 -671 for July 27.

The lower frequency band centers selected in Epoch II did not give good results, even though they cover more RRL $\alpha$ transitions. There is more artificial interference at frequencies below $5 \mathrm{GHz}$, and several of the zoom bands had to be discarded entirely. The conclusion for the subsequent SHRDS survey is that the best frequency placement is a compromise between the two shown in Figure 1. For the full survey, we chose to center our broad bands at 5.505 and $8.540 \mathrm{GHz}$. In this configuration, the SHRDS can observe 18 RRL $\alpha$ transitions simultaneously.

\section{Data Processing and Analysis}

Bandpass calibration, flux density calibration, and flagging were carried out with standard MIRIAD reduction techniques (Sault et al. 1995). After calibration, the zoom bands can be aligned in velocity and resampled to the same channel spacing and then averaged in order to improve the S/N. For Epoch I, we set the common channel spacing to $\Delta V=2.5 \mathrm{~km} \mathrm{~s}^{-1}$, and in Epoch II we use $2.3 \mathrm{~km} \mathrm{~s}^{-1}$ (Table 2). The resampling was done with the MIRIAD task UVAVER, which uses Fourier extension to change the channel step size. We average $u v$ spectra weighting by the continuum flux density of each baseline and each band. Longer baselines generally give weaker continuums, as most H II regions are partially resolved even with the H75 array. Any individual transitions that were polluted by RFI or had bad baseline ripples due to calibration problems were not included in the final average spectra.

Working with $u v$ plane data can blend together spectra from multiple objects within the primary beam. To separate individual sources or source components in a crowded field requires imaging. This analysis will follow when the full SHRDS survey data are available.

A Gaussian fit was made to the line profile in the average spectrum for each candidate. For lines with $\mathrm{S} / \mathrm{N}>3$, the Gaussian parameters are given in Table 3 and illustrated in Figures 2 and 3. Table 3 gives for each detected source, the source name in column 1. Column 2 has an $\mathrm{S}$ (for "Source Average") if the detection was made in the average of all RRL transitions or the Hn $\alpha$ number for sources bright enough to be detected in individual transitions. The following columns give the line center velocity (VLSR), line width (FWHM), continuum flux density $\left(S_{C}\right)$, spectral rms noise, peak line flux density $\left(S_{L}\right)$, electron temperature estimated from the line-tocontinuum ratios $\left(T_{e}\right)$, and the $\mathrm{S} / \mathrm{N}$. The $\mathrm{S} / \mathrm{N}$ is computed as (e.g., Lenz \& Ayres 1992):

$$
\mathrm{S} / \mathrm{N}=0.7\left(\frac{S_{L}}{\mathrm{rms}}\right) \sqrt{\frac{\mathrm{FWHM}}{\Delta V}}
$$



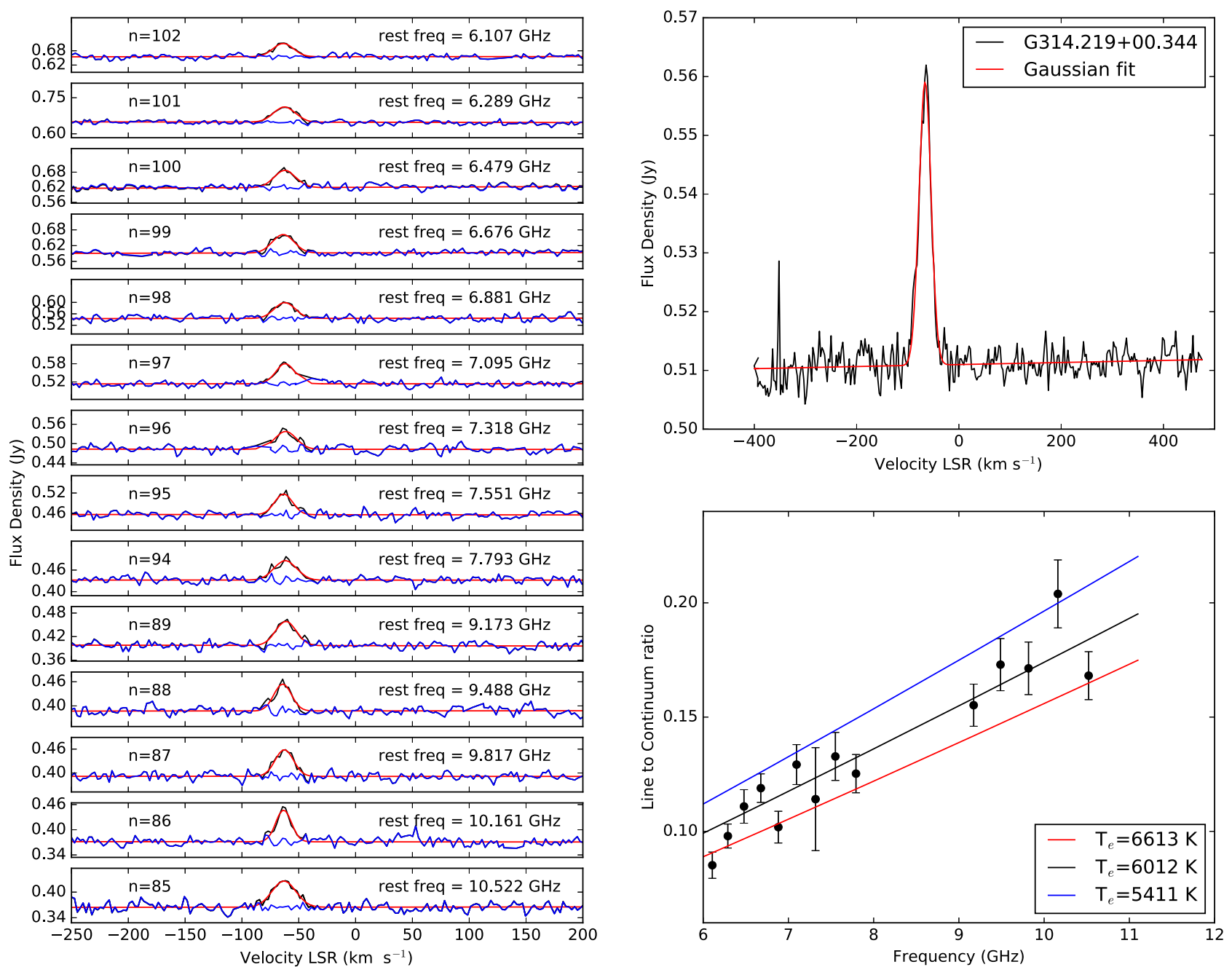

Figure 2. Spectra from a bright but previously unknown source. This figure shows the spectra for G314.219+00.344, which is a moderate to strong source, not previously detected in RRL emission. Individual RRL transitions are shown on the left panel, the overall average with Gaussian fit is shown on the upper right. The lower-right panel shows the line-to-continuum ratio for each RRL transition vs. frequency. Fitting these data with the assumption that both the line and continuum emission are optically thin gives an electron temperature $T_{e} \simeq 6000 \mathrm{~K}$ (black line). The red and blue lines correspond to increase and decrease by $10 \%$ of the best fit $T_{e}$, as indicated.

If a line was detected with $\mathrm{S} / \mathrm{N}$ greater than $\sim 15$ in the average spectrum, then each individual $\operatorname{Hn} \alpha$ transition was considered separately, again weighting the different baselines by their continuum flux. Gaussians were fitted to the average line profile for each transition. The results are listed as separate entries under each source name in Table 3. For these lines, the $\mathrm{S} / \mathrm{N}$ is simply the peak line flux divided by its error.

For lines sufficiently strong enough that their peak can be determined fairly accurately for each transition separately, it is possible to estimate the electron temperature, under the assumption that both the spectral line and continuum emission are optically thin. Whether or not this assumption holds depends on the emission measure. Typically for diffuse H II regions, the continuum is optically thin for frequencies above 3-5 GHz, but for ultra-compact and hyper-compact $\mathrm{H} \mathrm{II}$ regions, the continuum can be optically thick up to frequencies much higher than the $\mathrm{C} / \mathrm{X}$ band observed here (4-11 GHz). If the line and continuum are both optically thin and the level populations are in thermodynamic equilibrium with the electron kinetic temperature, $T_{e}$, then the line-to-continuum ratio, $S_{L} / S_{C}$, is

$$
\begin{aligned}
\frac{S_{L}}{S_{C}}= & 7 \times 10^{3}\left(\frac{\Delta V_{\mathrm{FWHM}}}{\mathrm{km} \mathrm{s}^{-1}}\right)^{-1}\left(\frac{\nu}{\mathrm{GHz}}\right)^{1.1} \\
& \times\left(\frac{T_{e}}{\mathrm{~K}}\right)^{-1.15}\left(1+\frac{N\left(\mathrm{He}^{+}\right)}{N\left(\mathrm{H}^{+}\right)}\right)^{-1}
\end{aligned}
$$

where $\Delta V_{\mathrm{FWHM}}$ is the line width (full width to half maximum, or 2.35 times $\sigma_{v}$ ), $\nu$ is the line rest frequency, and the ratio of column densities of $\mathrm{He}^{+}$to $\mathrm{H}^{+}$is taken as 0.09 , making the final term 1.09 (Quireza et al. 2006).

An interferometer telescope is particularly well suited to the measurement of $T_{e}$, because each baseline at each frequency measures $S_{L}$ and $S_{C}$ through the same spatial filter or fringe pattern. Although different baselines measure different continuum flux values, depending on the angular size and structure of the source and the projected baseline length, there is no need to determine a zero-level or overall offset to the continuum flux, as there is for single-dish surveys. In principal, every Hn $\alpha$ transition provides a separate measurement of $T_{e}$ by 

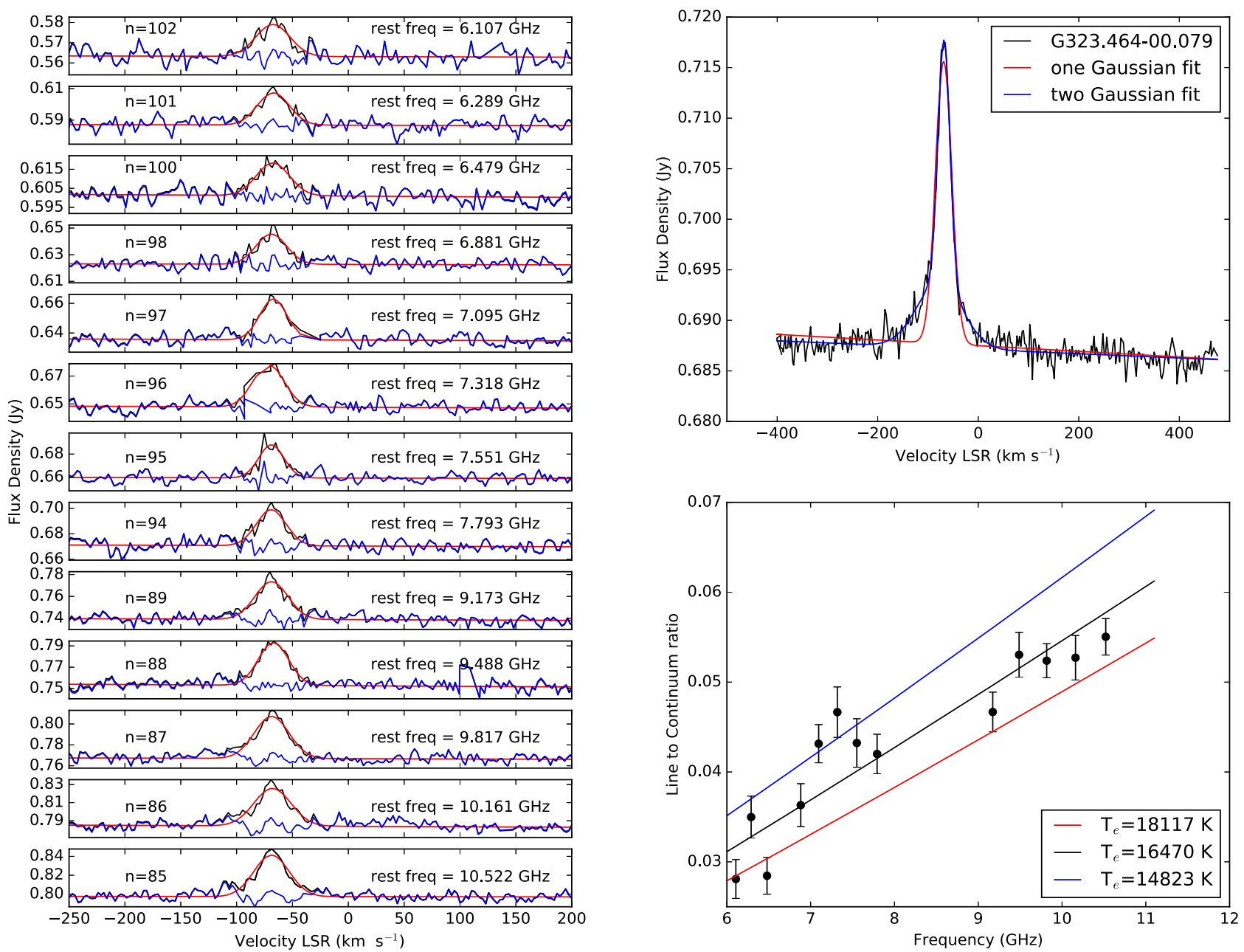

Figure 3. Spectra for a bright source that shows multiple line widths. This figure shows the spectra for G323.464-00.079, which is a strong source, previously detected by Murphy et al. (2010), with panel layout the same as in Figure 2. This hyper-compact H II region has a broad line profile that is not adequately fitted by a single Gaussian. This may indicate that there are two ionized regions with very different kinetic temperatures in the same $\mathrm{H}$ II region complex. The results of the twoGaussian fit are given in Table 3 and indicated by "S2" in column 2.

Equation (2) (column 8 in Table 3). A better estimate is given by fitting the line-to-continuum ratios for all lines to a single $T_{e}$ value. This is shown for the two sample sources on the lowerright panels of Figures 2 and 3.

The second example, G323.464-00.079, is a hyper-compact $\mathrm{H}$ II region that violates the condition of being optically thin in the continuum (Murphy et al. 2010). Unlike most of our detections, including G314.219+00.344 shown in Figure 2, the continuum flux of G323.464-00.079 increases rapidly with increasing frequency across the entire range of these observations. In this case, the electron temperature derived from the line-to-continuum ratios is an overestimate. Further study of this source using maps of the RRL emission may reveal whether the two Gaussian components correspond to separate regions of ionized gas.

Accurate values of the continuum and spectral line flux densities will require mapping and cleaning of the interferometer data. The full SHRDS survey achieves much better coverage of the $u v$ plane, as it uses two different array configurations and longer integrations at more and different hour angles. Thus, the values in columns 5 and 7 of Table 3 will be revised when better data become available. But the line to continuum ratios, and, hence, the electron temperatures, can be determined quickly from the $u v$ data alone, as is shown in the lower-right panels of Figures 2 and 3.

\section{Summary and Conclusions}

The SHRDS Pilot Study discovered 36 H II regions with RRLs, a detection rate of about $66 \%$. All known $\mathrm{H}$ II regions included in the observing schedule were also successfully redetected. More than one-third (15 out of 35) of the newly discovered H II regions are located in the outer Galaxy where existing catalogs of $\mathrm{H}$ II regions are not very complete. For the sources with RRL detections in the literature, the line strengths, center velocities, and line widths are in good agreement with published values. The rms of the differences in center velocities divided by their errors is 1.1 , and the rms of the differences in FWHM divided by their errors is 1.4. The only velocity parameter that differs from its corresponding value in the literature by more than $2 \sigma$ is the FWHM of the line in G295.748-00.207, which we find as $23.9 \pm 3.5 \mathrm{~km} \mathrm{~s}^{-1}$ versus Caswell \& Haynes (1987)'s value of $35 \mathrm{~km} \mathrm{~s}^{-1}$. Caswell \& Haynes (1987) do not give an error in the FWHM, but their channel spacing is $2.3 \mathrm{~km} \mathrm{~s}^{-1}$.

A surprising result of these pilot observations is the low detection rate for candidate $\mathrm{H}$ II regions in the third quadrant 
compared with those in the fourth quadrant of the Galaxy. This may be caused in part by a selection bias against sources with large angular sizes. The interferometer is not sensitive to brightness that is smoothly spread over angular sizes larger than about $6^{\prime}$, as noted in Section 3, paragraph 1 . Thus, nearby regions with large radii will show lower flux densities for the interferometer than they would to a single-dish telescope.

The main goal of these pilot experiments was to demonstrate the efficiency and sensitivity of the ATCA for detecting RRL emission from Galactic $\mathrm{H}$ II regions using the CABB system. The secondary objective was to determine the best placement for the $\mathrm{CABB}$ zoom bands, given the varying system temperature and interference environment of the ATCA and the typical emission spectra of a sample of $\mathrm{H}$ II region candidates. The result of the two epochs of observations indicates that the SHRDS should concentrate on the higher frequencies available with the $4 \mathrm{~cm}$ (C/X-band) receiver. These are generally easier to calibrate, more sensitive to RRLs from typical $\mathrm{H}$ II region candidates, and the resolution is better at shorter wavelengths.

The observing strategy for the SHRDS was also a subject of experimentation in the pilot project. For candidates near the detection threshold, an efficient strategy is to make many short observations at widely spaced hour angles, so as to get the best coverage of the $u v$ plane. These three- or four-minute integrations are not long enough to detect RRLs, but with 5-10 such "snapshots", a continuum map with fair to good dynamic range can be made. Based on the strength of the source(s) found on the continuum map, we can accurately estimate the expected RRL line strength. The total telescope time available can then be apportioned among the candidates so as to optimize the line detection rate.

This research has made use of of NASA's Astrophysics Data System, the SIMBAD database, and VizieR catalog access tool, CDS, Strasbourg, France. The National Radio Astronomy Observatory is a facility of the National Science Foundation operated under cooperative agreement by Associated Universities, Inc. The Australia Telescope Compact Array is part of the Australia Telescope National Facility, which is funded by the Australian Government for operation as a National Facility managed by CSIRO.

Software: ATCA observation scheduling tool (http://www. narrabri.atnf.csiro.au/observing/sched/cabb_nopw/), miriad (Sault et al. 1995), matplotlib (Hunter 2007).

\section{References}

Anderson, L. D., Armentrout, W. P., Johnstone, B. M., et al. 2015, ApJS, 221,26

Anderson, L. D., Bania, T. M., Balser, D. S., et al. 2014, ApJS, 212, 1

Anderson, L. D., Bania, T. M., Balser, D. S., \& Rood, R. T. 2011, ApJS, 194, 32

Balser, D. S. 2006, AJ, 132, 2326

Bania, T. M., Anderson, L. D., \& Balser, D. S. 2012, ApJ, 759, 96

Bania, T. M., Anderson, L. D., Balser, D. S., \& Rood, R. T. 2010, ApJL, 718, L106

Benjamin, R. A., Churchwell, E., Babler, B. L., et al. 2003, PASP, 115 953

Bock, D. C.-J., Large, M. I., \& Sadler, E. M. 1999, AJ, 117, 1578

Carey, S. J., Noriega-Crespo, A., Mizuno, D. R., et al. 2009, PASP, 121, 76

Caswell, J. L., \& Haynes, R. F. 1987, A\&A, 171, 261

Churchwell, E., Babler, B. L., Meade, M. R., et al. 2009, PASP, 121, 213

Condon, J. J., Cotton, W. D., Greisen, E. W., et al. 1998, AJ, 115, 1693

Helfand, D. J., Becker, R. H., White, R. L., Fallon, A., \& Tuttle, S. 2006, AJ, 131,2525

Hoare, M. G., Purcell, C. R., Churchwell, E. B., et al. 2012, PASP, 124, 939

Hunter, J. D. 2007, CSE, 9, 90

Lenz, D. D., \& Ayres, T. R. 1992, PASP, 104, 1104

McClure-Griffiths, N. M., Dickey, J. M., Gaensler, B. M., et al. 2005, ApJS, 158,178

Misanovic, Z., Cram, L., \& Green, A. 2002, MNRAS, 335, 114

Murphy, T., Cohen, M., Ekers, R. D., et al. 2010, MNRAS, 405, 1560

Purcell, C. R., Hoare, M. G., Cotton, W. D., et al. 2013, ApJS, 205, 1

Quireza, C., Rood, R. T., Bania, T. M., Balser, D. S., \& Maciel, W. J. 2006, ApJ, 653, 1226

Sault, R. J., Teuben, P. J., \& Wright, M. C. H. 1995, in ASP Conf. Ser. 77, Astronomical Data Analysis Software and Systems IV, ed. R. A. Shaw, H. E. Payne, \& J. J. E. Hayes (San Francisco, CA: ASP), 433

Shaver, P. A., McGee, R. X., Newton, L. M., Danks, A. C., \& Pottasch, S. R. 1983, MNRAS, 204, 53

Umana, G., Trigilio, C., Franzen, T. M. O., et al. 2015, MNRAS, 454, 902

Wilson, W. E., Ferris, R. H., Axtens, P., et al. 2011, MNRAS, 416, 832

Wright, E. L., Eisenhardt, P. R. M., Mainzer, A. K., et al. 2010, AJ, 140, 1868 